\title{
MAGMA-SMC: The Molecular Cloud Survey of the SMC
}

\section{Erik Muller ${ }^{1}$, Tony Wong ${ }^{2}$, Annie Hughes ${ }^{3}$, Jürgen Ott $^{4}$, Jorge L. Pineda ${ }^{5}$ and the MAGMA collaboration}

\author{
${ }^{1}$ NAOJ, Chile Observatory, 2-21-1 Osawa, Mitaka, Tokyo 181-8588, Japan; email \\ erik.muller@nao.ac.jp. ${ }^{2}$ Astronomy Department, University of Illinois, Urbana, IL 61801, \\ USA. ${ }^{3}$ Max-Planck-Institut für Astronomie, Königstuhl 17, D-69117, Heidelberg, Germany. \\ ${ }^{4}$ NRAO, Socorro, NM, USA. ${ }^{5}$ JPL, CIT, 4800 Oak Grove Drive, Pasadena, CA 91109, USA.
}

\begin{abstract}
We present a brief summary and description of the upcoming ${ }^{12} \mathrm{CO}(1-0)$ Magellanic Mopra Assesment (MAGMA) SMC survey data releaseł. The MAGMA-SMC survey has sampled $100 \%$ of the known $\mathrm{CO}$ in the SMC (at $\sim 33^{\prime \prime}$ resolution; $12 \mathrm{pc}$ at D $=60 \mathrm{kpc}$ ). Having explored $522 \times 10^{3}$ square parsecs throughout the SMC with $695^{\prime} \times 5^{\prime}$ fields, to a sensitivity of $\sim 150 \mathrm{mK}$, we apply the CLOUDPROPS (Rosolowsky \& Leroy 2006) cloud-search algorithm optimized for low $\mathrm{S} / \mathrm{N}$ data, to detect more than $30 \mathrm{CO}$ clouds with virial masses between $10^{3}-10^{4} \mathrm{M}_{\odot}$, mean radii $\sim 5 \mathrm{pc}$ and $0.3-0.9 \mathrm{~km} \mathrm{~s}^{-1}$ velocity width. Typical brightness temperatures are $\sim 1 \mathrm{~K} \mathrm{~T}_{m b}$. All detected molecular regions are associated with at least one $24 \mu \mathrm{m}$ compact emission source. Smoothing rarely increases the total detected $\mathrm{CO}$ flux, implying the $\mathrm{CO}$ emission is typically confined to small spatial scales. As recent dust maps of the SMC imply extended $\mathrm{H}_{2}$ mass, the apparent compact nature of the $\mathrm{CO}$ population indicates some departures from the canonical Galactic $X_{\mathrm{CO}}$-factor in the low-metallicity and relatively un-evolved ISM of the SMC.
\end{abstract}

Keywords. ISM: molecules — ISM: clouds - Magellanic Clouds

\section{References}

Bolatto, A. D., Simon, J. D., Stanimirović, S., et al. 2007, ApJ, 655, 212

Rosolowsky, E. \& Leroy, A. 2006, PASP, 118, 590

$\ddagger$ http://alma.mtk.nao.ac.jp/ erik/MAGMA-SMC

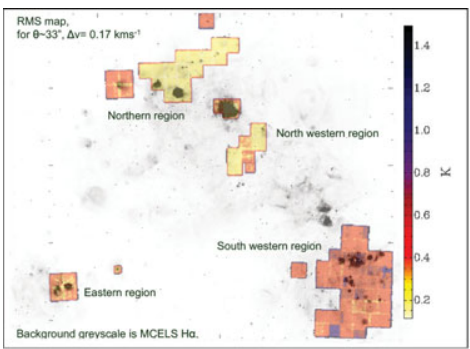

(Left) RMS map of sampled regions, overlaid on $\mathrm{H} \alpha$ (MCELS; priv. comm. F. Winkler). (Below) Three panels showing detected CO emission associated with four prominent nebulae: N76, N36, N12 and N22, overlaid as contours on 3-color images (RGB: $24 \mu \mathrm{m}$ ( $\left.\mathrm{S}^{3} \mathrm{MC}\right), \mathrm{H} \alpha$ (MCELS), $160 \mu \mathrm{m}$ ( $\mathrm{S}^{3} \mathrm{MC}$; Bolatto et al. 2007). The leftmost image shows integrated $\mathrm{CO}$ as identified by CLOUDPROPS, after smoothing to a $98^{\prime \prime}$ beam. The two right images are at the nominal resolution of $33^{\prime \prime}$.
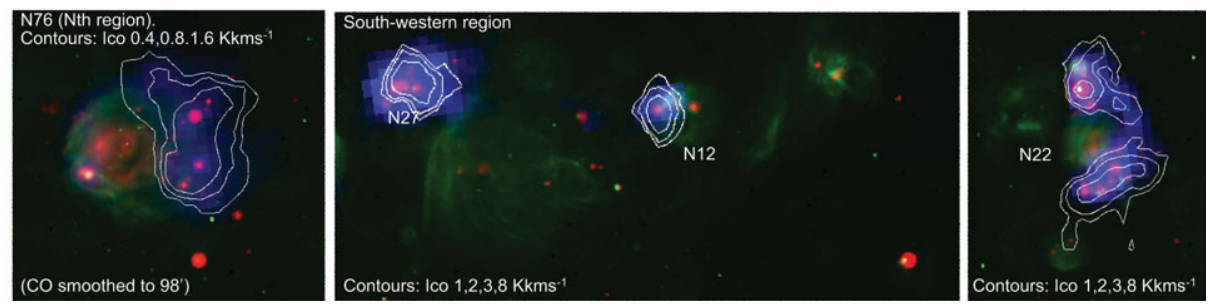